\title{
High-Dose-Rate 192Ir Brachytherapy Dose Verification: A Phantom Study
}

\author{
Alireza Nikoofar ${ }^{1}$; Zohreh Hoseinpour ${ }^{2}$; Seied Rabi Mahdavi ${ }^{3}$; Hadi Hasanzadeh ${ }^{4, *}$; Mostafa \\ Rezaei Tavirani ${ }^{5}$ \\ ${ }^{1}$ Department of Radiation Oncology, Iran University of Medical Sciences, Tehran, IR Iran \\ 2 Department of Medical Physics, Semnan University of Medical Sciences, Semnan, IR Iran \\ 3 Department of Medical Physics, Iran University of Medical Sciences, Tehran, IR Iran \\ ${ }^{4}$ Cancer Research Center \& Department of Medical Physics, Semnan University of Medical Sciences, Semnan, IR Iran \\ ${ }_{5}$ Cancer Research Center \& Department of Medical Physics, Semnan University of Medical Scienc
Proteomics Research Center, Shahid Beheshti University of Medical Sciences, Tehran, IR Iran \\ ${ }^{*}$ Corresponding author: Dr Hadi Hasanzadeh, Cancer Research Center \& Department of Medical Physics, Semnan University of Medical Sciences, Semnan, IR Iran. Tel: +98-2333451336, \\ E-mail: hasanzadeh.h@semums.ac.ir
}

Received: October 24, 2014; Revised: November 16, 2014; Accepted: December 14, 2014

\begin{abstract}
Background: The high-dose-rate (HDR) brachytherapy might be an effective tool for palliation of dysphagia. Because of some concerns about adverse effects due to absorbed radiation dose, it is important to estimate absorbed dose in risky organs during this treatment. Objectives: This study aimed to measure the absorbed dose in the parotid, thyroid, and submandibular gland, eye, trachea, spinal cord, and manubrium of sternum in brachytherapy in an anthropomorphic phantom.

Materials and Methods: To measure radiation dose, eye, parotid, thyroid, and submandibular gland, spine, and sternum, an anthropomorphic phantom was considered with applicators to set thermoluminescence dosimeters (TLDs). A specific target volume of about $23 \mathrm{~cm}^{3}$ in the upper thoracic esophagus was considered as target, and phantom planned computed tomography (CT) for HDR brachytherapy, then with a micro-Selectron HDR $\left({ }^{192} \mathrm{Ir}\right)$ remote after-loading unit.

Results: Absorbed doses were measured with calibrated TLDs and were expressed in centi-Gray (cGy). In regions far from target ( $\geq 16 \mathrm{~cm})$ such as submandibular, parotid and thyroid glands, mean measured dose ranged from 1.65 to $5.5 \mathrm{cGy}$. In closer regions $(\leq 16 \mathrm{~cm})$, the absorbed dose might be as high as 113 cGy.

Conclusions: Our study showed similar depth and surface doses; in closer regions, the surface and depth doses differed significantly due to the role of primary radiation that had imposed a high-dose gradient and difference between the plan and measurement, which was more severe because of simplifications in tissue inhomogeneity, considered in TPS relative to phantom.
\end{abstract}

Keywords: Esophageal Cancer; Brachytherapy; Phantom

\section{Background}

Esophageal cancer is the eighth most common cancer in the world and fourth common cause of death, which occurs often in thorax (1), with median survival of typically less than six months and $80 \%$ of mortalities related to progressive local disease (2). Conventional treatment of esophageal cancer includes surgery, chemotherapy, stent-placement, external radiotherapy, and brachytherapy. Brachytherapy is one of the main components of modern radiotherapy (3) in which radioactive sources are temporarily or permanently inserted into tumor. The Iridium-192 $\left({ }^{192} \mathrm{Ir}\right)$ is the most commonly used source in the clinical practice because of its high specific activity and short half-life. Many studies have suggested that the high-dose-rate (HDR) brachytherapy is an effective tool for palliation of dysphagia (4). Patients with esophageal stricture could be treated initially with external radiation therapy or chemotherapy and then, receive brachytherapy to complete treatment (5). Brachytherapy has been increasingly delivered at high dose-rates, with a dose of 12 Gy or more per hour. Dysphagia improvement has been reported in $50 \%$ of patients. The complication rate after single-dose brachytherapy is low (20\%) and mainly includes fistula formation, mild retrosternal pain, and radiation esophagitis. Persistent/recurrent dysphagia following single-dose brachytherapy has been most commonly caused by tumor persistence (15\%), tumor recurrence (35\%), and benign stricture formation (5\%) (6). Some adverse effects are observed with this treatment including dry and sore throat, respiratory problems, thyroid disorders, and pain similar to heart burn; this might be due to high dose transfer, particularly in HDR brachytherapy. Adverse effects depend mainly on the dose and type of radiation in which toxic dose 50 (TD50) is defined as 20 Gy for thyroid (7), maximum dose received by the spinal cord should not be more than $45 \mathrm{~Gy}(8)$, and mean dose to parotid and submandibular glands should not be more than 24.2 and 46.9 Gy, respectively $(9,10)$. Dosimeter at these high-

Copyright (C) 2015, Iranian Journal of Cancer Prevention. This is an open-access article distributed under the terms of the Creative Commons Attribution-Non Commercial 4.0 International License (http://creativecommons.org/licenses/by-nc/4.0/) which permits copy and redistribute the material just in noncommercial usages, provided the original work is properly cited. 
risk organs could prevent adverse effects and is initiated by transferring high dose to these organs and inhibits creation of secondary cancers, which included surface in vivo measurements on the body or in-depth measurements in anatomical phantoms designed to simulate the structure of the human body $(11,12)$. Numerous studies have suggested several dosimeters such as thermoluminescence dosimeters (TLDs), radiochromic film, and diode and ionization chamber $(13,14)$. Diode and ionization chamber were not very suitable because of their finite dimensions and TLD was more suitable and reliable to be used in brachytherapy and external radiation therapy (14). Moreover, TG-43 methods have a tendency to underestimate dose to bone, especially the ribs (15). The ${ }^{192}$ Ir radionuclide emits high-energy gamma rays with energies up to $1.4 \mathrm{MeV}$ that easily penetrate through patient's body. Dickler et al. (16) have shown the need to consider OARs in the evaluation and comparison of brachytherapy sources. However, they have not considered radiation dose to organs located outside the treatment volume where photon energy determines the dose. Clinical studies evaluating the adverse effects caused by irradiating healthy organs are needed so that physicians have better understanding when HDR ${ }^{192} \mathrm{Ir}$ might benefit a patient.

\section{Objectives}

In this study, the dose delivered to the parotid, thyroid, and submandibular gland, eye, trachea, spinal cord, and manubrium of sternum in brachytherapy were measured using TLD in an anthropomorphic phantom.

\section{Materials and Methods}

\subsection{Brachytherapy and HDR Treatment Unit}

The micro-Selectron HDR was remote after-loading unit, a treatment system developed by Flexitron International. The TPS used the American Association of Physicists in Medicine (AAPM), Task Group 43 (TG-43) formalism for dose calculation, which includes different methods of optimization of the treatment's dose distribution such as geometrical/graphical optimization and adjustment of dwell positions/times. The ${ }^{192}$ Ir source in this unit has an active length of $3.5 \mathrm{~mm}$ and active diameter of $0.6 \mathrm{~mm}$. The source is enclosed in a cylindrical stainless steel capsule of external diameter $1.1 \mathrm{~mm}$ and length $5.0 \mathrm{~mm}(14,17,18)$ (Figure 1). The 48FR esophagus applicator with length of $50 \mathrm{~cm}$ and diameter of $1.6 \mathrm{~cm}$ was inserted in esophagus lumen of phantom, which was used to place dummy source in-place to take computed tomographic (CT) scans of thorax for treatment planning. In this study, the prescribed dose per fraction was $5 \mathrm{~Gy}$ at $1 \mathrm{~cm}$ from the surface of the esophageal applicator with the treatment length including the tumor plus a $2 \mathrm{~cm}$ margin at both ends (4).

\subsection{Calibration of Dosimeters}

The TLDs (LiF-100, $3.1 \times 3.1 \times 0.9 \mathrm{~mm}$ square chips) were selected (19) and annealed before use in order to achieve better stability of their sensitivity and lower fading. The annealing was performed at $400^{\circ} \mathrm{C}$ for one hour and was followed by fast cooling and subsequent annealing at $80^{\circ} \mathrm{C}$ for 24 hours (Atash 1200 Exiton Crop, Iran). Subsequently, elemental correction coefficients $\left(\mathrm{ECC}_{\mathrm{i}}\right)$ were calculated by exposure to a dose of $200 \mathrm{cGy}$ with $6 \mathrm{MV}$ photons and were applied to each TLD as ECC $_{\mathrm{i}}=<\mathrm{R}>$ | $\mathrm{R}_{\mathrm{i}}$ where $<\mathrm{R}>$ was the mean signal of all dosimeters and Ri was the individual signal of each TLD (Harshaw TLD Reader, model 3500, the United States) (Figure 2). The calibration curve of TLDs was obtained by exposing TLDs to doses ranging from zero to $250 \mathrm{cGy}$ in Plexiglas slab phantom (depth, $3 \mathrm{~cm}$; distance, $97 \mathrm{~cm}$ ).

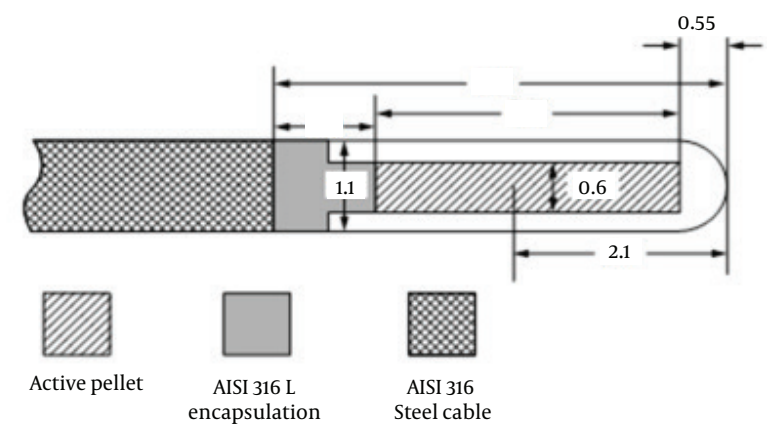

Figure 1. High-Dose-Rate ${ }^{192}$ Ir Brachytherapy Source Type Designs

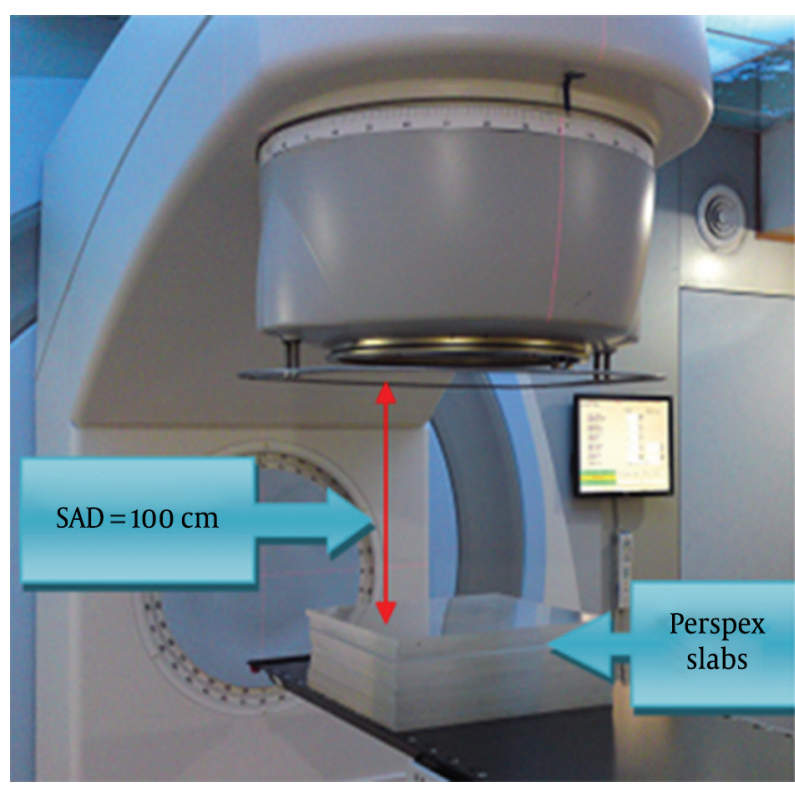

Figure 2. Setup of Thermoluminescence Dosimeters Calibration 


\subsection{Phantom Structure}

The phantom used in this study was an anthropomorphic phantom constructed from natural bone and mixture of paraffin wax with sodium chloride as impurity for soft tissue, with effective atomic number and electron density of $6.57 \times 10^{23}$ and $3.36 \times 10^{23}$ electrons/g, respectively. Tissue substitutes for lungs were two spongy woods with similar dimensions and density to lungs. Several applicators were provided to locate TLD in several depths and an esophageal lumen was considered for entrance of esophagus applicator $(20,21)$ (Figure 3).

\subsection{Phantom Dosimetric Measurements}

In order to plan the phantom for considered treatment, CT scans were acquired from the phantom with applicator placed into phantom. During CT scanning, external markers were used to avoid any mismatch between the planned and the treated phantom set up (Figure 4). All CT data were sent to the Flexiplan 3D TPS (Pars radiotherapy center, Tehran, Iran) and then the ${ }^{192}$ Ir sources were remotely loaded into the phantom while TLDs were placed at considered locations (10 on surface and six in depths) (Figure 4). Dwelling times and positions of the single ${ }^{192} \mathrm{Ir}$ source were planned to deliver 5 Gy to the specific target volume with length of $4 \mathrm{~cm}$ and volume of $23 \mathrm{~cm}^{3}$ in the upper thoracic esophagus lumen. Target volume was CTV with $1 \mathrm{~cm}$ distal and proximal margins. The considered locations for TLDs were eye, right parotid (skin surface and 1-cm depth), left parotid (skin surface and 1-cm depth), left submandibular, right submandibular, left thyroid (skin surface and 1-cm depth), and right thyroid glands (skin surface and 1cm depth), trachea (skin surface and 4-cm depth), manubrium of sternum, spine (inside the phantom and skin surface).

\section{Results}

The calibration curve with its linear regression equation, which related the collected charge in TLDs to radiation-absorbed dose, is shown in Figure 5.

Radiation doses received by organs at risk and organs near the transit rout of source in the phantom were measured with calibrated TLDs and then expressed in cGy (Table 1). In addition, the calculated dose from TPS is shown in a separate column for comparison.

\section{Discussion}

In this study, the appropriate brachytherapy plan was considered to determine dwelling times and the positions of ${ }^{192}$ Ir sources. The accuracy of TPS was evaluated using TLD dosimeters and was compared with TPS calculated values. Commercially available TPS use different methods to calculate clinical dose-rate distributions such as single-source data as tables or mathematical formulas, which need certain coefficients (19). There were some differences between results of TPS and TLD measurements

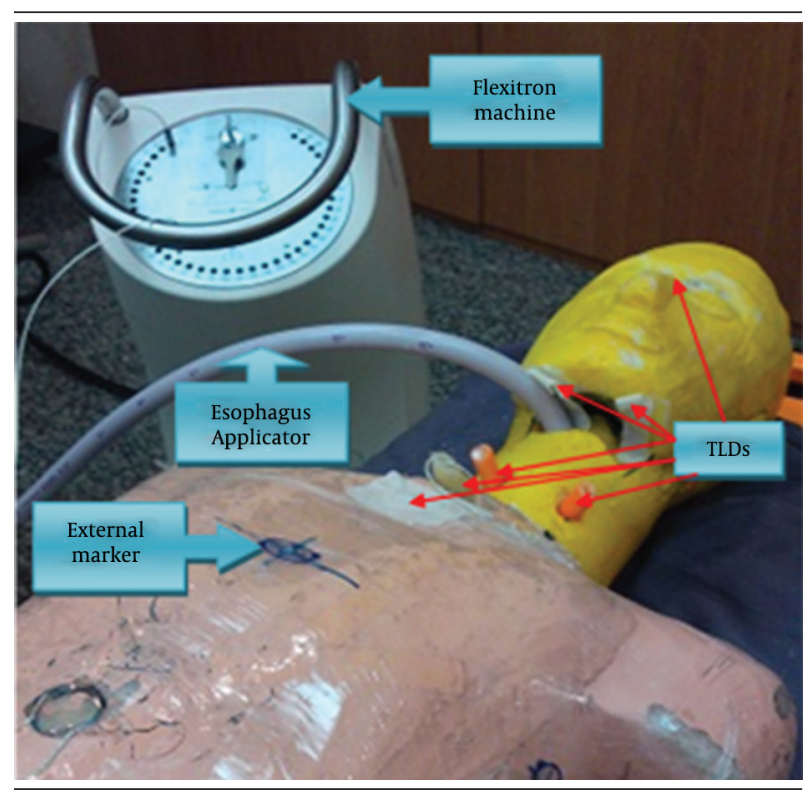

Figure 3. Anthropomorphic Phantom Connected to the Flexitron HighDose-Rate Machine With Esophagus Applicator and Thermoluminescence Dosimeters Placed in Arbitrary Positions

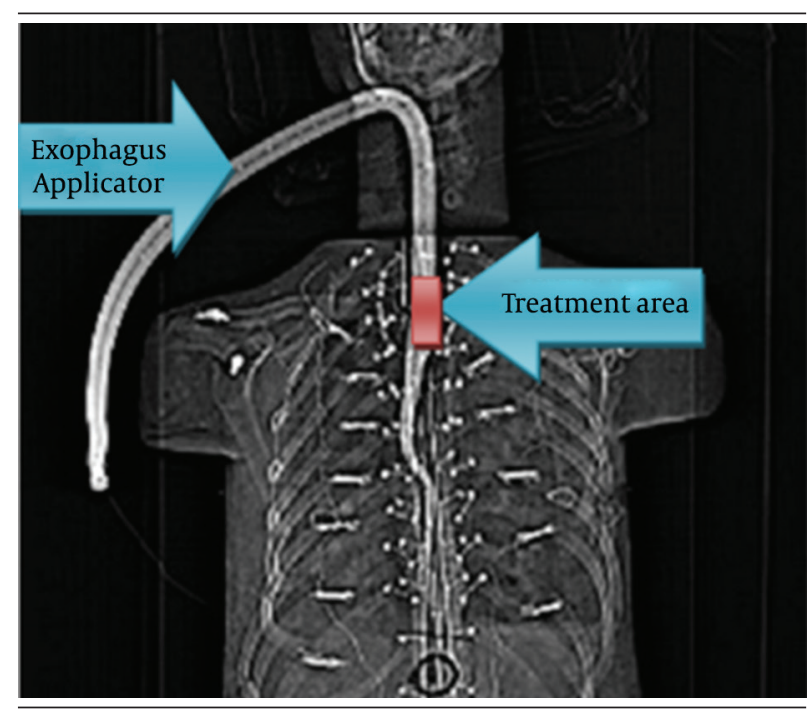

Figure 4. Computed Tomographic Scan of Phantom With Esophagus Applicator

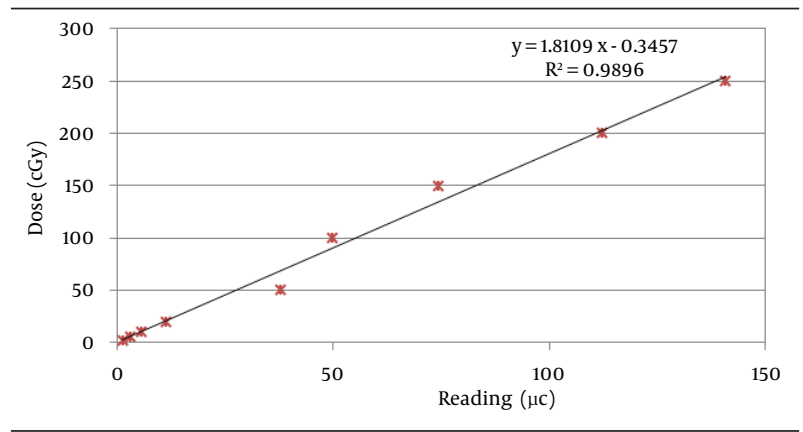

Figure 5. Calibration Curve for Thermoluminescent Dosimeters 
NikoofarA et al.

\begin{tabular}{|c|c|c|c|}
\hline \multirow[t]{2}{*}{ Organ } & \multicolumn{2}{|c|}{ Absorbed Dose, cGy } & \multirow[t]{2}{*}{ Mean Distance From Target, cm } \\
\hline & Measured & Calculated & \\
\hline Eye & $1.65 \pm 0.04$ & $N A^{a}$ & $>25$ \\
\hline \multicolumn{4}{|l|}{ Parotid } \\
\hline Depth & $2.73 \pm 0.76$ & 2 & 19 \\
\hline Surface & $2.00 \pm 0.72$ & 2 & 17 \\
\hline Submandibular & $5.51 \pm 0.81$ & 4 & 16 \\
\hline \multicolumn{4}{|l|}{ Thyroid } \\
\hline Depth & $6.16 \pm 0.93$ & 20 & 8 \\
\hline Surface & $7.95 \pm 1.81$ & 17 & 7 \\
\hline \multicolumn{4}{|l|}{ Trachea } \\
\hline Depth & $113.37 \pm 0.30$ & 134 & 8 \\
\hline Surface & $6.60 \pm 0.34$ & 16 & 3.5 \\
\hline \multicolumn{4}{|l|}{ Spine } \\
\hline Depth & $79.59 \pm 2.91$ & 100 & 4 \\
\hline Surface & $9.10 \pm 0.60$ & 20.1 & 8 \\
\hline Manubrium of Sternum & $10.87 \pm 2.66$ & 15.6 & 9.5 \\
\hline
\end{tabular}

that might be due to the TPS calculation algorithm in which tissue inhomogeneity was not considered as existed in phantom. This problem was more severe in places with more primary radiation, e.g., thyroid, trachea, spine, and sternum, and TPS had overestimated absorbed dose. In other words, dose distribution around sources not only was depended on distance from source and its size, but also was related to the tissues absorption and scattering $(22,23)$. Moreover, the geometry function in dose calculation formalism had neglected scattering and attenuation. It provided an inverse square-law correction based on approximate model of the spatial distribution of activity in the source (19). In places where scattered radiation was dominant, the difference was negligible although some differences might result from TLD inherent limitations. The dose-rate contributions assumed that all tissues in and around the implant were water equivalent (at least $5 \mathrm{~cm}$ of water-equivalent material surrounded the point of calculation) (19). This might affect the dose calculation in closer regions to the source; as seen in Table 1 , the TPS overestimated the doses to thyroid, trachea, spine and manubrium of sternum. The severity of this effect was more in the vicinity of source.

In addition, our results showed that increasing distance from dwelling source position leaded to a rapid fall-off in dose. Trachea and spine were very close to the dwelling source position, but they received different dose; this might be due to high gradient as their corresponding dosimeters were placed at slightly different sites (between $\mathrm{T} 2$ and $\mathrm{T} 3$ for spine and in front of $\mathrm{T} 1$ and $\mathrm{T} 2$ for trachea). The measured dose at parotid, thyroid and submandibular glands might be due to both transit dose and scattered radiation. Furthermore, in places near to the source, decrease in the dose might be due to inverse square law; but at larger distances tissue absorption caused a reduction in dose and scattering increased it. At larger distances, dose distribution was affected by both absorption and scattering phenomena and a deviation from the inverse square law might cause a deviation of about $20 \%$ in absorbed dose, depending on the source's energy $(22,23)$. Marinello et al. (24) recommended always taking hyperdose sleeve, which was the volume receiving a dose equal to or greater than twice the reference dose, into account. One unavoidable problem with intraluminal brachytherapy was the steep gradient of dose distribution between the radioactive source and the surface mucosa. Narrower applicators (diameter, $8 \mathrm{~mm}$ ) tended to deliver high doses to the mucosa, which was associated with an increased risk of esophageal fistulas (25). In this study, we used 48 FR esophagus applicator with 1.6-cm diameter, so there was no problem with hyperdose sleeve.

Yorozu et al. (25) have shown that increasing incidence of esophageal ulcers is related to the prescribed dose in brachytherapy, and doses more than 16 Gy lead to an improvement in local tumor control and significant risk of ulceration. Severe ulcers have commonly encountered when large fractions as high as 5 Gy or more, or a total dose of 15 Gy were employed with a narrow tube applicator. In our research, prescribed dose was 5 Gy or total dose was 10 Gy in two fractions; therefore, it decreased probability of fistula formation.

According to our results, there was no difference between depth and surface dose in parotid and thyroid glands; it was only possible if absorbed dose to these 
organs was due to scattered radiation when radiation source had been placed locally (20) and neglecting scattering and attenuation in dose calculation and considering an inverse square-law correction (19).

This study was performed to estimate the absorbed dose to parotid, thyroid, and submandibular glands, spine, trachea, sternum, and eye. The high accuracy of this type of in vivo dosimetry is not always needed. Our results showed that sternum dose was less than the dose Uniyal et al. (26) obtained by film dosimetry, which could be due to many reasons such as length of treatment area, dwelling position, dwelling time, and place of dosimeters. The absorbed dose to trachea was obtained from surface and in-depth measurements. Therefore, one could estimate the dose of trachea in depth from skin dosimetry, which was beneficial for patient dosimetry. Moreover, it could be used for quality control of TPS.

Intraluminal brachytherapy is a useful tool for treatment of esophageal cancer with definitive and palliative goals. Dosimetric studies on phantoms are applicable and useful for training brachytherapy systems and have been considered as a valuable tool for QA program as well as verification of TPS, even for non-standard conditions. Between all dosimeters, TLD is relatively easy and accurate in vivo measurement tool to evaluate dose delivery in brachytherapy systems. The results of in vivo measurements showed that the absorbed dose at distant positions were due to scattered radiation, which caused relatively similar depth and surface; however, in closer positions for defined targets, there were some effects of primary radiation and depth and surface doses were different. Some organs such as eyes and parotid, submandibular, and thyroid glands received very low doses, which did not show major effects on patients' quality of life. Other organs such as trachea, spine, and manubrium of sternum received approximately the same dose as was calculated by TPS.

\section{Acknowledgements}

This study was conducted by a research grant provided by the Research Deputy of Semnan University of Medical Sciences, Semnan, Iran, and kind collaboration of Pars Radiotherapy Center.

\section{Authors' Contributions}

Hadi Hasanzadeh, Alireza Nikoofar, and Seied Rabi Mahdavi designed the study, gathered and analyzed the data, and wrote the manuscript. Zohreh Hoseinpour contributed to study design, phantom irradiation, treatment planning, and writing and overall correction of the manuscript.

\section{Conflict of Interest}

The authors made no disclosures.

\section{References}

1. Chen J, Zhu J, Pan J, Zhu K, Zheng X, Chen M, et al. Postoperative radiotherapy improved survival of poor prognostic squamous cell carcinoma esophagus. Ann Thorac Surg. 2010;90(2):435-42.

2. Rosenblatt E, Jones G, Sur RK, Donde B, Salvajoli JV, Ghosh-Laskar S, et al. Adding external beam to intra-luminal brachytherapy improves palliation in obstructive squamous cell oesophageal cancer: a prospective multi-centre randomized trial of the International Atomic Energy Agency. Radiother Oncol. 2010;97(3):488-94.

3. Hoskin PJ, Bownes P. Innovative technologies in radiation therapy: brachytherapy. Semin Radiat Oncol. 2006;16(4):209-17.

4. Devlin PM.Brachytherapy:applications and technique. Philadelphia,PA: Lippincott Williams \& Wilkins; 2007.

5. Vuong T, Szego P, David M, Evans M, Parent J, Mayrand S, et al. The safety and usefulness of high-dose-rate endoluminal brachytherapy as a boost in the treatment of patients with esophageal cancer with external beam radiation with or without chemotherapy. Int J Radiat Oncol Biol Phys. 2005;63(3):758-64.

6. Siersema PD, Vleggaar FP. Esophageal strictures, tumors, and fistulae: alternative techniques for palliating primary esophageal cancer. Tech Gastrointest Endosc. 2010;12(4):203-9.

7. Alterio D, Jereczek-Fossa BA, Franchi B, D'Onofrio A, Piazzi V, Rondi E, et al. Thyroid disorders in patients treated with radiotherapy for head-and-neck cancer: a retrospective analysis of seventythree patients. Int J Radiat Oncol Biol Phys. 2007;67(1):144-50.

8. Khan FM. Treatment planning in radiation oncology. Lippincott Williams \& Wilkins; 2011.

9. Jellema AP, Doornaert P, Slotman BJ, Leemans CR, Langendijk JA. Does radiation dose to the salivary glands and oral cavity predict patient-rated xerostomia and sticky saliva in head and neck cancer patients treated with curative radiotherapy? Radiother Oncol. 2005;77(2):164-71.

10. Roesink JM, Moerland MA, Battermann JJ, Hordijk GJ, Terhaard $\mathrm{CH}$. Quantitative dose-volume response analysis of changes in parotid gland function after radiotherapy in the head-and-neck region. Int J Radiat Oncol Biol Phys. 2001;51(4):938-46.

11. Lindskoug BA. Clinical applications of thermoluminescent dosimetry. Strahlentherapie. 1985;161(2):91-5.

12. Aschan C. Applicability of thermoluminescent dosimeters in X-ray organ dose determination and in the dosimetry of systemic and boron neutron capture radiotherapy Helsink. Helsinki: University of Helsinki; 1999.

13. Gambarini G, Borroni M, Grisotto S, Maucione A, Cerrotta A, Fallai $\mathrm{C}$, et al. Solid state TL detectors for in vivo dosimetry in brachytherapy. Appl Radiat Isot. 2012;71 Suppl:48-51.

14. Uniyal SC, Sharma SD, Naithani UC. A dosimetry method in the transverse plane of HDR Ir-192 brachytherapy source using gafchromic EBT2 film. Phys Med. 2012;28(2):129-33.

15. Mille MM, Xu XG, Rivard MJ. Comparison of organ doses for patients undergoing balloon brachytherapy of the breast with HDR 192Ir or electronic sources using monte carlo simulations in a heterogeneous human phantom. Med Phys. 2010;37(2):662-71.

16. Dickler A, Kirk MC, Seif N, Griem K, Dowlatshahi K, Francescatti $\mathrm{D}$, et al. A dosimetric comparison of MammoSite high-doserate brachytherapy and Xoft Axxent electronic brachytherapy. Brachytherapy. 2007;6(2):164-8.

17. Williamson JF, Li Z. Monte Carlo aided dosimetry of the microselectron pulsed and high dose-rate 192Ir sources. Med Phys. 1995;22(6):809-19.

18. Baltas D, Sakelliou L, Zamboglou N. The physics of modern brachytherapy for oncology. CRC Press; 2010.

19. Rivard MJ, Coursey BM, DeWerd LA, Hanson WF, Huq MS, Ibbott GS, et al. Update of AAPM Task Group No. 43 Report: A revised AAPM protocol for brachytherapy dose calculations. Med Phys. 2004;31(3):633-74.

20. Hasanzadeh H, Sharafi A, Allah Verdi M, Nikoofar A. Assessment of absorbed dose to thyroid, parotid and ovaries in patients undergoing Gamma Knife radiosurgery. Phys Med Biol. 2006;51(17):4375-83. 


\section{NikoofarA et al.}

21. Hasanzadeh $\mathrm{H}$, Abedelahi A. Introducing a simple tissue equivalent anthropomorphic phantom for radiation dosimetry in diagnostic radiology and radiotherapy. J Param Sci. 2011;2(4):25-9.

22. Dimmick LC, Bass B, Waksman R, Moscovitch M. Estimation of radiation dose received during treatment of in-stent restenosis using ionizing radiation. Cardiovasc Revasc Med. 2005;6(1):24-31.

23. Venselaar JL, van der Giessen PH, Dries WJ. Measurement and calculation of the dose at large distances from brachytherapy sources: Cs-137, Ir-192, and Co-60. Med Phys. 1996;23(4):537-43.
24. Marinello G, Pierquin B, Grimard L, Barret C. Dosimetry of intraluminal brachytherapy. Radiother Oncol. 1992;23(4):213-6.

25. Yorozu A, Dokiya T, Oki Y, Suzuki T. Curative radiotherapy with high-dose-rate brachytherapy boost for localized esophageal carcinoma: dose-effect relationship of brachytherapy with the balloon type applicator system. Radiother Oncol.1999;51(2):133-9.

26. Uniyal SC, Sharma SD, Naithani UC. Dosimetric verification of a high dose rate brachytherapy treatment planning system in homogeneous and heterogeneous media. Phys Med. 2013;29(2):171-7. 